J3eA, Journal sur l'enseignement des sciences et technologies de l'information et des systèmes, Volume 4, Hors-Série 2, 37 (2005)

DOI : http://dx.doi.org/10.1051/bib-j3ea:20057037

(C) EDP Sciences, 2005

\title{
Mu.Psi - Fibula, un kit DSP simple et efficace pour l'enseignement et l'industrie
}

$$
\text { J.M. Ory }
$$

Centre de Recherche en Automatique de Nancy (CRAN)

École Supérieure des Sciences et Technologies de l'Ingénieur de Nancy (ESSTIN)

Nancy, France 


\title{
Mu.Psi - Fibula, un kit DSP simple et efficace pour l'enseignement et l'industrie.
}

\author{
Jean-Marie ORY \\ CRAN (Centre de Recherche en Automatique de Nancy) \\ ESSTIN (Ecole Supérieure des Sciences et Technologies de l'Ingénieur de Nancy) \\ ory@esstin.uhp-nancy.fr
}

\section{Résumé}

Afin de répondre aux besoins d'efficacité en enseignement du traitement des signaux en temps réel par DSP (processeur numérique de signaux), nous avons créé une carte DSP industrielle Mu.Psi ainsi qu'un environnement logiciel de développement rapide, FIBULA fondé sur l'interconnexion de blocs fonctionnels hiérarchisés. Dans l'industrie, cette plate-forme de développement permet de créer des maquettes temps réel bien plus rapidement qu'avec les outils traditionnels. Nous utilisons en particulier cet environnement matériel - logiciel pour enseigner les transmissions numériques d'une manière originale.

Mots clefs : DSP, DSP56300, plate-forme, développement, bloc fonctionnel, signal, temps réel

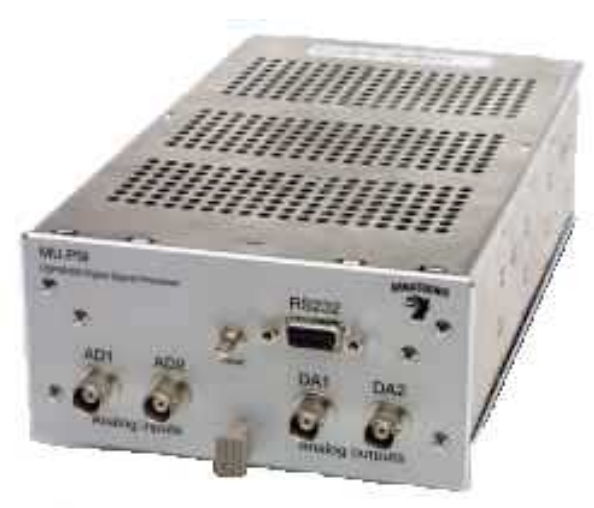

Fig. 1 Carte Mu.Psi en module HAMEG ${ }^{\circledR}$

\section{INTRODUCTION}

Lorsqu'un débutant veut aborder les processeurs de signaux (DSP), il se heurte souvent à la mise en œuvre d'outils matériels et logiciels extrêmement lourds qui nécessitent au préalable la lecture d'une abondante documentation.

Avec le kit DSP Mu.psi (Fig. 1) et l'environnement FIBULA (Fig. 2), nous avons voulu aller à l'essentiel: traiter des signaux en temps réel.

Le langage textuel à blocs fonctionnels interconnectés est extrêmement simple. La prise en mains du kit pour un débutant ne prend en général que quelques minutes.

Le produit est fabriqué et distribué par la Sté Arnatronic (www.arnatronic.com).

\section{LA CARTE Mu.Psi}

En créant la carte Mu.Psi, nous avons tenté de répondre à un besoin de l'industrie pour des applications orientées instrumentation ou contrôle régulation. Construite autour d'un DSP56309

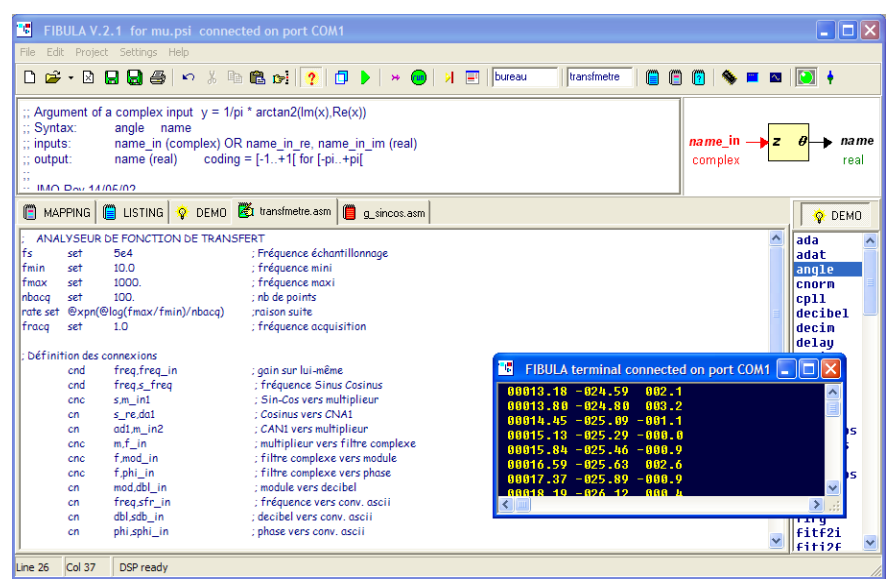

Fig. 2 L'environnement de développement FIBULA

Motorola (100 MIPS, arithmétique fractionnaire 2448 bits) elle possède 2 entrées et 2 sorties analogiques 12 bits. L'acquisition instantanée sur 2 voies se fait sans filtre anti-repliement à une fréquence d'échantillonnage programmable entre $1 \mathrm{~Hz}$ et $1 \mathrm{MHz}$. Cela permet, entre autres, des démonstrations très pédagogiques du repliement spectral, et permet l'échantillonnage de signaux HF. Toutes les connexions logiques sont accessibles à l'aide de connecteurs fiabilisés; on y trouve (Fig. 3):

- 1 port parallèle de 16 bits

- 2 ports série synchrones rapides (ESSI)

- 1 port série asynchrone avec interface RS232

- 3 Timers, 4 entrée d'interruptions

- 1 port JTAG / OnCE pour le debug matériel

La carte Mu.Psi est disponible, soit nue, soit montée dans un module Hameg® embrochable, avec connections analogiques BNC en face avant où elle peut être associée à d'autres instruments (Fig. 1). 


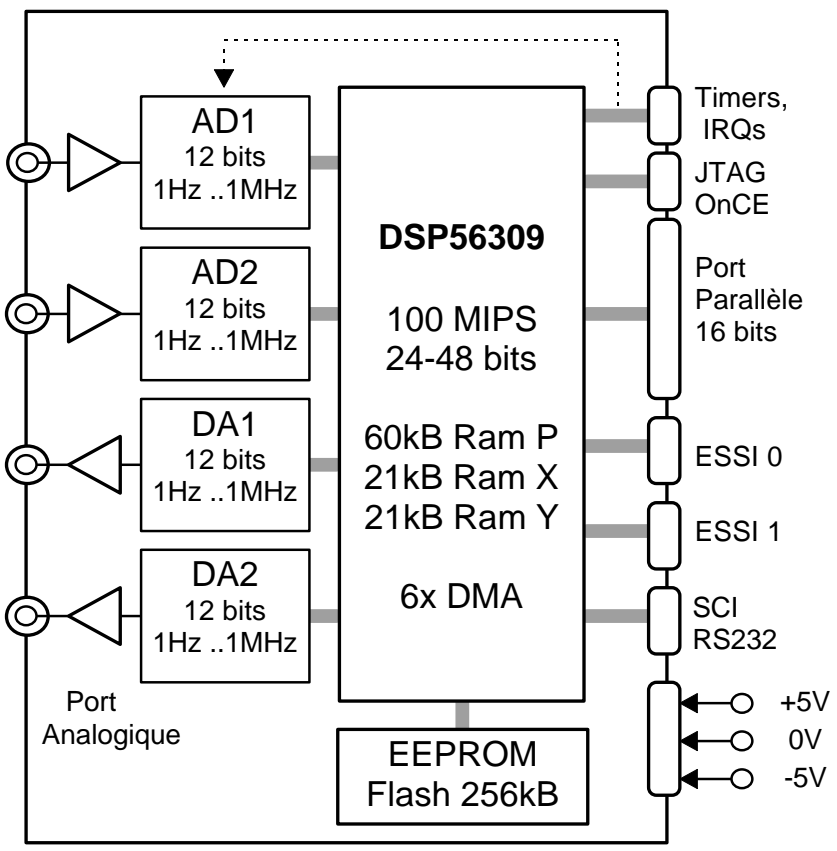

Fig. 3 Schéma de la carte Mu.Psi

\section{L'ENVIRONNEMENT LOGICIEL FIBULA}

FIBULA est un compilateur de langage textuel à "blocs fonctionnels" tournant sous Win32. Le programmeur décrit de manière très compacte le flux des données (connexions) et l'ordre d'exécution des blocs. Il dispose pour cela d'une large bibliothèque ouverte de fonctions écrites en assembleur sous forme de macros. Il peut utiliser ces fonctions telles quelles, ou en faire des versions modifiées, ou encore créer de nouvelles fonctions à partir de fonctions existantes. Le code machine obtenu est quasi optimal. La clef de cette performance tient à une technique originale de connexions virtuelles à zéro cycle machine. De plus, FIBULA permet sans limitations de créer des blocs de niveaux hiérarchiques supérieurs à l'aide de plusieurs blocs interconnectés, cela également avec un coût de zéro cycle machine.

FIBULA affiche une fenêtre qui représente l'ensemble des blocs fonctionnels disponibles avec la syntaxe correspondante et un graphisme montrant la nomenclature et le type des connexions disponibles. Chaque bloc est accompagné d'un programme de démonstration (Fig.2). On peut également visualiser le jeu d'instructions assembleur du DSP avec les syntaxes correspondantes.

FIBULA permet de travailler soit uniquement à partir de blocs fonctionnels, soit en assembleur pur, soit de mixer l'assembleur et les blocs fonctionnels.

La barre d'outils offre de nombreuses facilités dont:

- Un mode d'exécution bloc par bloc avec affichage des valeurs aux connexions,

- Le couplage au simulateur logiciel Motorola pour pas-à-pas machine détaillé,
- La visualisation du listing, du mapping, des informations de timing,

- L'ouverture d'un terminal pour communications série,

- Un oscilloscope virtuel 8 voies, avec récupération des images et des données,

- Une abondante aide en ligne (30Mo de documents pdf)

\section{LA PROGRAMMATION PAR BLOCS FONCTIONNELS}

\subsection{Un exemple pratique}

Dans l'exemple qui suit, proposons-nous de créer un analyseur de fonction de transfert (Fig. 4).

Un signal sinusoïdal $\cos (\omega t)$ dont la fréquence varie selon une loi exponentielle est appliqué à l'entrée du système inconnu. La sortie du système est multipliée par la sinusoïde complexe $\mathrm{e}^{\mathrm{j} \omega \mathrm{t}}$, et le résultat complexe est filtré passe-bas, de manière à extraire la composante en phase et la composante en quadrature. A partir de ces deux composantes, on calcule la puissance (carré du module) que l'on exprime en $\mathrm{dB}$, et la phase exprimée en degrés. (En procédant ainsi, on évite les erreurs dues à la présence d'une éventuelle composante continue).

La figure 5 représente le code complet de cette application.

Chaque bloc possède un identificateur de fonction (par exemple g_sincos pour "générateur sinuscosinus"), et un nom unique qui représente une instance particulière de ce bloc (par exemple "s").

La progression des fréquences selon une loi géométrique est obtenue par un simple gain freq qui est connecté sur lui-même (un bouclage rétroactif engendre automatiquement un délai $\mathrm{z}^{-1}$ ) Sa sortie représente la fréquence imposée au générateur sinus-cosinus $\mathbf{s}$. Le signal $\mathbf{s}$ est considéré comme complexe. Sa partie réelle est transformée en tension analogique par le convertisseur da1, laquelle est injectée dans le système inconnu. La sortie analogique du système est échantillonnée et quantifiée par le convertisseur ad1. Le multiplieur m réalise la multiplication complexe du signal recueilli ad1 par le signal s.

Les termes du produit en $2 \omega$ sont éliminés par le passe-bas complexe du $1^{\text {er }}$ ordre f. Ensuite on calcule le module mod et la phase phi du résultat. Le module est ensuite exprimé en décibels grâce au bloc dbl.

Enfin, à l'aide des blocs strd, on transforme les grandeurs machine en chaînes de caractères décimaux de manière à ce que la fréquence apparaisse en $\mathrm{Hz}$, le gain soit en décibels, et le déphasage soit en degrés. Les chaînes décimales sont concaténées avec séparateurs et caractères de fin de ligne avant d'être transmis sur le port asynchrone RS232 à l'aide du bloc snds. Ce bloc utilise un canal DMA, cela évite de consommer du temps CPU dans la boucle d'attente. 


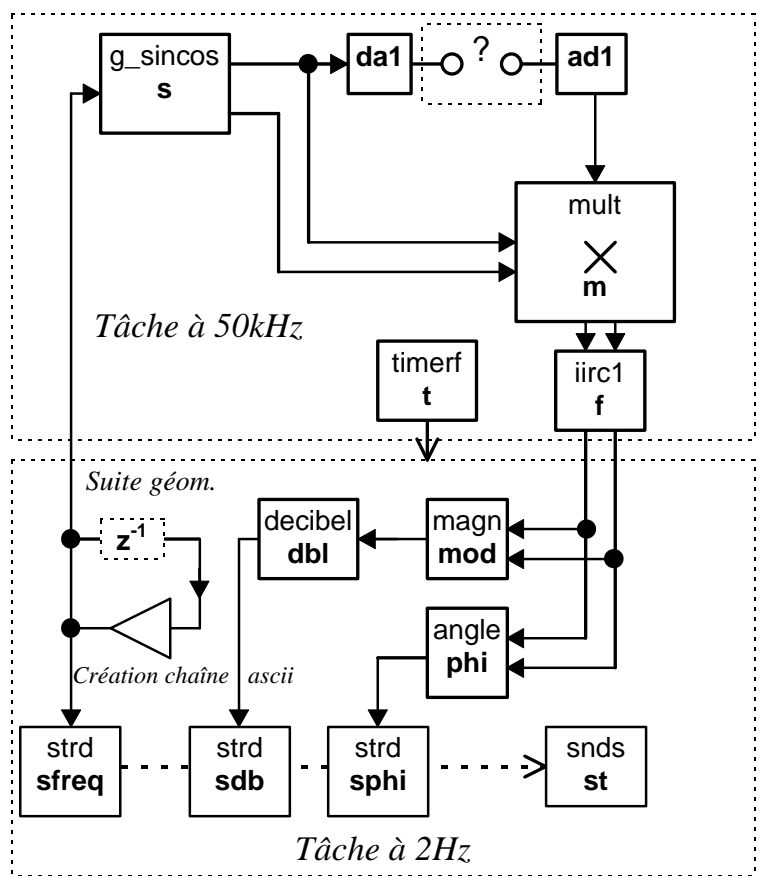

Fig. 4 Exemple d'application: transféromètre

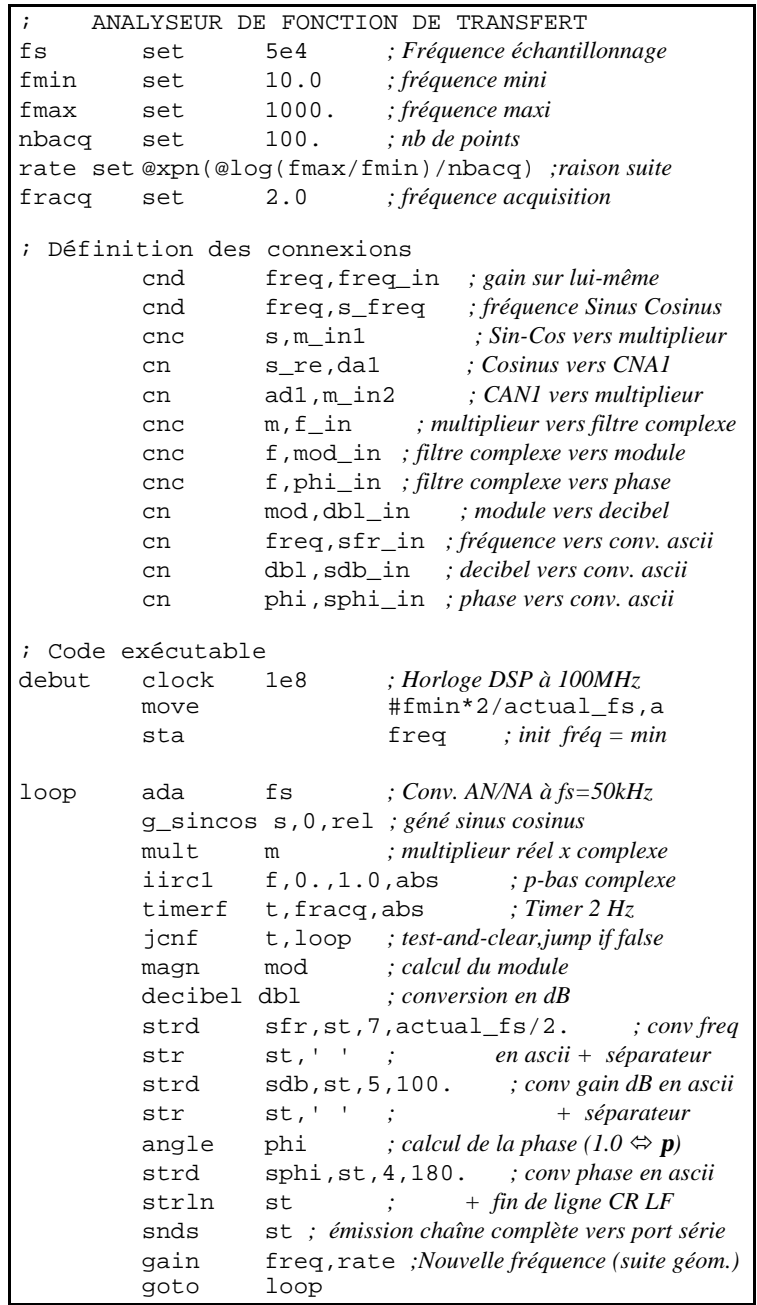

Fig. 5 Code de la figure 4
Dans le schéma de la figure 4, la partie supérieure est exécutée à la fréquence d'échantillonnage de $50 \mathrm{kHz}$, tandis que la partie inférieure qui consiste à créer et envoyer la chaîne de caractères n'est exécutée que toutes les demi-secondes.

Le timer $\mathrm{t}$ associé à un branchement conditionnel réalise ce sous-échantillonnage à $2 \mathrm{~Hz}$.

Le code représenté en figure 5 se compose de 3 parties:

1 / Constantes et paramètres de l'application

2 / Connexions entre blocs

3 / Partie exécutable

Les connexions créent les variables et déterminent leurs types. Ainsi, cn est une connexion simple 24 bits, cnd est une connexion double précision (48 bits), et cnc est une connexion complexe $2 \times 24$ bits.

\subsection{Quelques aspects du langage à blocs fonctionnels}

\section{Nomenclature}

A chaque instance d'un bloc est associé un identificateur unique de 1 à 512 caractères. Lorsqu'un bloc est constitué de sous-blocs, les noms des sous-blocs commencent tous par le nom du bloc principal augmenté d'un tiret. Ainsi, il est possible d'accéder à n'importe quelle variable enfouie. Par exemple, dans un récepteur radio numérique, la variable rf_mixer_osc_freq représente la valeur de la fréquence de l'oscillateur utilisé dans le mélangeur de l'étage haute fréquence.

\section{Gestion du temps et des fréquences}

L'horloge du DSP est réglable entre $12 \mathrm{kHz}$ et 150MHz par paramétrage des diviseurs du PLL. Par exemple, pour obtenir une fréquence de $100 \mathrm{MHz}$, il suffit d'écrire:

\section{clock 1 e8}

La valeur exacte obtenue (fdsp $=101,58 \mathrm{MHz})$ est mémorisée pour les calculs de temps et fréquences. La fréquence d'échantillonnage et d'acquisition $\mathrm{AD}$ et $\mathrm{DA}$ est réglable de $5 \mathrm{~Hz}$ à $1 \mathrm{MHz}$. Par exemple, pour acquérir à $100 \mathrm{kHz}$, on écrit

$$
\text { ada } 1 \text { e5 }
$$

De même, la fréquence exacte obtenue (ici: actual_fs $=100,178 \mathrm{kHz}$ ) sert dans la suite à calibrer toutes les fonctions du temps ou de la fréquence.

Ainsi, lors de calculs de filtres, lignes à retard, etc.

.., on pourra:

- Soit exprimer les temps en échantillons et les fréquences relativement à Fs/2 ( option rel ),

- Soit exprimer les fréquences en Hertz et les temps en secondes (option $a b s$ ).

La compilation de la macro "ada" génère un message de la forme:

Available DSP cycles between samples $=984$ 
Le programmeur peut alors vérifier si son algorithme est exécutable en temps voulu. Pour cela il utilise les macros TIC et TOC afin de réaliser un chronométrage matériel:

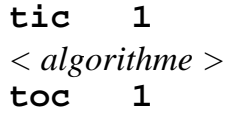

Le temps mesuré est exprimé en cycles machine dans la fenêtre "terminal" de Fibula.

Dans les applications de type multi-fréquence, seule la fréquence la plus élevée résulte d'une interruption matérielle; les autres sont obtenues à l'aide de timers logiciels. Ces timers sont soit mono-coup, soit périodiques; ils utilisent soit le comptage d'échantillons soit l'intégration numérique et fournissent en sortie une variable booléenne.

Exemple:

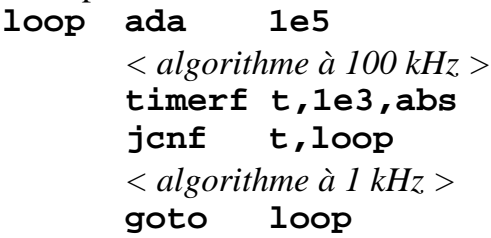

timerf est un timer logiciel périodique défini par une fréquence absolue. A chaque échéance, il lève la variable booléenne t.

jcnf est un saut conditionnel avec remise à zéro de la variable t.

Si la tâche basse fréquence est de durée importante, on fait exécuter la tâche haute fréquence sous interruption:

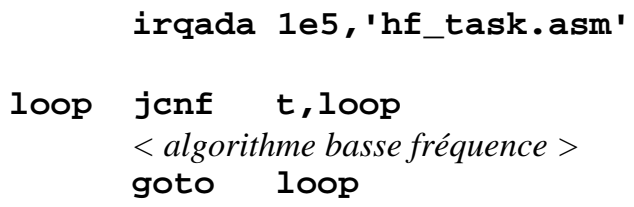

La tâche haute fréquence est enregistrée dans un fichier séparé, la sauvegarde et la restauration du contexte altéré est automatiquement prise en charge.

\section{QUELQUES APPLICATIONS ORIGINALES}

\subsection{Applications industrielles}

Commande non linéaire auto-adaptative de postes de soudure TIG (L'Air Liquide)

Commande de hacheurs courants forts (Pont-àMousson)

Traitement TR d'images CCD linéaires (SNCF)

Imagerie RMN en champ faible

Capteur de vitesse à corrélation optique

\subsection{Applications pédagogiques}

Bases du traitement du signal échantillonnage et repliement spectral mesures, filtres, analyse spectrale.

Signaux aléatoires, traitements statistiques
Puissance et spectre du bruit de quantification Histogramme d'une somme de $\mathrm{n}$ variables uniformes.

Calcul en TR de propriétés statistiques: valeur RMS, Facteur de crête, kurtosis, skewness

Systèmes auto-adaptatifs et non linéaires:

Annulation d'écho ( algorithme LMS)

Réseaux de neurones à apprentissage en TR

Simulation de systèmes chaotiques, avec visualisation des attracteurs.

\subsection{Un module TP d'introduction aux transmissions numériques}

Pour mettre en place une série de TP qui ne soit pas constituée de simples démonstrations, nous avons créé une bibliothèque contenant les principales briques pour construire une application de transmission numérique. Les étudiants doivent connecter les blocs fonctionnels entre eux et les paramétrer correctement.

Une première séance est consacrée aux transmissions en bande de base, avec les problèmes de synchronisation d'horloge, de canal bruité à bande limitée (diagramme de l'œil), et de suppression d'échos (LMS). Ensuite sont vus les différents types de modulations (ASK, FSK, PSK, QAM) et montrons dans chaque cas l'influence du bruit et celle de la limitation de bande dans le canal. Une expérience très spectaculaire consiste à réaliser un récepteur radio $\mathrm{AM}$ grandes ondes avec visualisation simultanée du spectre, à l'aide d'un simple barreau ferrite dont l'enroulement est connecté sur une entrée analogique.

Nous montrons également les techniques de multiplexage temporel, fréquentiel, et par codes pseudo aléatoires orthogonaux (CDMA).

\section{CONCLUSIONS}

La carte Mu.Psi a beaucoup évolué dans le sens de la fiabilité et de la robustesse. Son utilisation dans plusieurs applications industrielles à courants forts le prouve.

Le nouvel environnement de développement FIBULA remporte l'adhésion des étudiants qui l'apprécient pour son interactivité et sa facilité de mise en œuvre.

La plate-forme Mu.Psi - Fibula constitue l'outil idéal pour réaliser très rapidement des prototypes temps réel, projets ou maquettes $\mathrm{R} \& \mathrm{D}$.

\section{Références:}

Site du fabricant / distributeur:

www.arnatronic.com 